surprised to learn at the end of the narration, that the patient received no benefit from the treatment adopted. Even had the result been otherwise, it would in no wise alter the facts; because I grant, that all my cases where anæmia, etc., existed, might have been benefited by iron and counter-irritants; but could they ever have been cured by them ? Before I knew as much as I now do about the nature of these cases, I have whitewashed many by chalybeates, rest, and banishing mothers from their homes; but let them only return to the old regime, and they invariably lapse back to that condition whence they started.

I am free to confess that Dr. Bennet's method of practice may be abused, and discredit cast upon the legitimate use of the speculum by its indiscriminate employment. But because some are found base enough to abuse medical confidence, is it therefore to be abandoned, and a good system decried?

4, Shaw Street, Liverpool, Nov. 21, 1851.

\title{
MORBID VASCULARITY OF THE LINING MEMBRANE OF THE FEMALE URETHRA.
}

By GEORGE T. GREAM, M.D., late one of the Medical Officers to Queen Charlotte's Lying-in Hospital.

UNDER the name of "Vascular Tumour of the Orifice of the Meatus Urinarius", this affection was first described by Sir Charles Clarke, in his valuable work on the Diseases of Women; a work giving evidence of the highly practical knowledge of that author, and which, although published more than thirty-five years ago, may still be considered as one of the best guides to the diagnosis of this class of maladies.

He writes : "There is in most women a degree of projection round the orifice of the meatus urinarius, and from this part sometimes the tumour arises, to which the above name of the vascular tumour of the meatus urinarius has been applied." I have ventured to refer to this affection under another name, because my own experience, confirmed by that of others, tends to show that it does not always appear as a tumour, but that it may be present under other forms, accompanied by the same general as well as local symptoms.

Dr. Ashwell has correctly described the disease, but he speaks of it more especially as a tumour, and states that it is rarely seen after the cessation of the menses. I am led to think that he is mistaken in this respect, for I have witnessed the disease as often in elderly women as in the young.

Dr. Meigs, of Philadelphia; has alluded to the complaint in his volume on Females and their Diseases; but he merely refers to it as a portion of the lining membrane of the urethra, "hypertrophied and inflamed, which may be readily cut off." His observations, however, are contained in a very few lines, in which the importance of the disease is altogether overlooked.

I am not aware that any other authors have noticed the affection at all; but certainly none have regarded it with that consideration which 
it calls for, when we consider the suffering attending it, and its liability to return, unless properly treated and entirely removed. The fact that it is one of those diseases which do not frequently come under the notice of medical men, renders the circumstance of its being so little alluded to by authors especially remarkable.

It presents itself under three different forms : the first, most likely, being the incipient stage of the second; and the second, the beginning of the third. But this is only conjecture; for the opportunity of proving it has not yet been afforded to me. It may be present as a simple vascularity of the lining membrane of the urethra, without any elevation whatever, extending some little distance towards the bladder; the membrane itself being highly florid in colour, and extremely tender when touched, or during the passage of the urine. This is the usual character of the disease, when it is confined within the canal; but Sir Charles Clarke relates the case of a patient in St. Bartholomew's Hospital, in whose urethra there was a tumour of a scarlet colour, nearly filling up the canal. The occurrence of a tumour, however, within the urethra is unusual. This is probably owing to the pressure of the sides of the canal preventing the elevation of the dilated vessels, and to the passage of the urine having a similar effect.

When the vascularity is within the urethra, no morbid appearances present themselves externally; but if the symptoms call attention to the part, and the lining membrane is exposed by making pressure around the meatus, the highly florid appearance will at once be detected.

The second form in which the disease appears, is that of a flattened vascular spot, with but slight elevation, surrounding the orifice of the urethra, highly florid in colour, and exquisitely tender when touched : it is so little elevated, that it can scarcely be called a tumour. The redness extends from it into the canal for some little distance, but the membrane within, although florid in appearance, is quite smooth on its surface; whereas the external spot of vascularity is slightly granulated, because it is not modified by pressure from the sides of the urethra.

In the third stage, the disease consists of a distinct tumour, granulated, and attached, sometimes by a broader base, sometimes by a narrow one, and, in some instances, even by a slender pedicle to the side of the urethra, or just externally to it ; and, in almost all cases, some dilated vessels will be seen extending from its base to within the urethral canal.

When there is an actual prominent tumour, the local pain and the constitutional symptoms are greatly increased in severity. In some cases, the peculiar scarlet colour of the part has attracted the notice of the patient; but in many instances, particularly when the vascularity is within the urethra, not only has the actual seat of the disease escaped her observation, but it has also been overlooked by her medical attendant, who has referred to the uterus as the diseased organ, has stated that its cervix was inflamed or ulcerated, and caustic has sometimes for weeks, or months, been applied, without affording the least advantage to the patient.

This vascular disease is not at all to be considered as similar to an affection situated in the same parts, having its origin in a varicose state of the veins, which causes some uneasiness and is accompanied by a mucous discharge, but which does not produce the same acute 
suffering nor the great constitutional disturbance, nor is the appearance the same. In the vascular disease in question the blood contained in the vessels is arterial, while in the venous enlargement it is dark coloured, and the distended veins have the same appearance which veins have in other parts of the body when in a varicose condition. Attention is first called to the vascular disease, by an uneasy sensation at the lower part of the body, and pain passing down the thighs; and pain when urine is voided, or when the part is touched; slight bleeding also occurs occasionally, owing to the rupture of some dilated vessel, whose covering is always much attenuated. There may be frequent desire to pass urine; and walking causes great suffering; while accompanying these symptoms, there is always copious mucous discharge, which is excessive when the disease appears in the form of a tumour. Owing to which, as well as to the constant uneasiness and frequent acute suffering, the patient becomes emaciated and weak, and it is surprising to find so many and such severe symptoms arising from a disease whose extent is confined within such limited bounds; but there is clear evidence that it does produce them in the fact that, immediately upon the destruction of the vascular spot, or even on its partial removal, a comparative freedom from the symptoms is at once enjoyed.

Upon a digital examination of the vagina being made, great tenderness is experienced by the patient at the vaginal orifice, and still more if pressure is made towards the pubes; and bleeding from this part will almost always be induced by the examination. These symptoms necessarily call for further investigation, and the nature of the disease becomes apparent.

The only mode of cure is the destruction of the entire congeries of vessels; and if the smallest part of it is left, the disease will most certainly return. It has been customary to employ excision with scissors, and afterwards to apply potassa fusa, or to use the latter alone for the removal of the complaint. A ligature has also been recommended as a means of removal; but there are objections to both these modes of treatment, which those who have used them will, I am confident, admit. In order that the scissors may be employed, the vagina must be held open by an assistant; but the spot to be excised is so covered by the pubes that it cannot be sufficiently exposed to ensure the due performance of the operation, and the potassa fusa applied to the bleeding surface is effectual only in those cases in which the disease is very superficial, and by itself this caustic always fails to destroy the part entirely; hence we have a return of the complaint, and a repetition of a most painful operation.

If the disease is within the urethra, these means of cure are totally inapplicable. If the ligature is employed, it may in some cases prove efficient; but in others it would be impossible to effect a cure by means of it, as the part affected could not all be enclosed.

Having several times been called upon to treat cases which had been before apparently cured (by myself and others) by the means mentioned above, I was led to think of another mode of treatment which would be more successful; and it occurred to me that the application of strong nitric acid, in the manner adopted by Mr. Henry Lee for the destruction of hæmorrhoids (and which proves so successful), would be equally applicable to the vascularity of the female urethra. 
Sufficient time has now elapsed since its application in three instances, and yet there is not the least inclination to a return of the disease in them; and as in others, although more recently treated, there is like-. wise no such disposition, I am able to speak with some confidence respecting this mode of cure. Its comparative advantages consist in the acid being minutely applicable to each individual part of the affected spot, which it has the power of entirely destroying, whether within the urethra or outside of it; in its producing no fear in the patient as does the anticipation of an operation by a cutting instrument; and in the pain which it causes very quickly subsiding. Dr. Ashwell observes: "The main trouble we encounter in the treatment of these tumours, is their tendency to reappear. If they are snipped off with scissors, and the part allowed spontaneously to heal, there is every probability that it will repullulate and cause the same symptoms. If these growths are not moveable and attached by a pedicle, I have found that the diligent application of nitrate of silver freely applied over and around them, will eventually get rid of them : but the process of destruction is tedious and attended with great agony. It is generally indeed necessary to apply opium to the part after application, and to soothe the patient by some morphia or extract of hyoscyamus at night." Now there is no such necessity after the application of nitric acid; but the patient complains of no pain after a few minutes have elapsed, and she is able to walk about without inconvenience.

But there is a difficulty in exposing the part sufficiently, and in preventing the sides of the vagina from collapsing too soon after the application of nitric acid; and this is overcome by the use of a speculum, invented, I believe, by $\mathrm{Mr}$. Hilton, for the removal of hæmorrhoidal excrescences. A portion of the side of the speculum, extending nearly to its internal extremity, can be removed after its introduction into the vagina, and if this part of it is just under the pubes, the spot of vascularity will project into the tube ; but should only the lining membrane of the urethra be vascular, it will be readily exposed by pressing the speculum firmly towards the pubes against the surrounding parts : and the acid can be applied while the pressure is kept up.

A small rod of glass, or a piece of hard wood in the form of the stick of a camel's hair pencil, is the best thing with which to apply the acid; and this should be held to the part for about a minute, care being taken that each enlarged portion of the vessels is completely destroyed, and in about three or four minutes the pain attending it ceases, and the speculum can be removed. It will be better to examine the part in about four days from the time of the application of the acid, and it often will be found healed with no trace of the complaint left. More frequently it presents an unhealed sore, but an absence of the disease. If, however, there be any vessel remaining having the peculiar scarlet colour, it should be again touched with nitric acid, otherwise the symptoms will rapidly return,

Hertford Street, May Fair, January 1852. 